\title{
Integration of Heterogeneous Systems via DICOM and the Clinical Image Access Service
}

\author{
RA Moreno ${ }^{1,2}$, SS Furuie ${ }^{1}$ \\ ${ }^{1}$ Heart Institute (InCor), University of São Paulo Medical School, São Paulo, Brazil \\ ${ }^{2}$ Polytechnic School(POLI), University of São Paulo, São Paulo, Brazil
}

\begin{abstract}
In this paper we present a proposal for the integration of DICOM Structured Reporting (DICOM SR) and the Clinical Image Access Service (CIAS). The advantage of this approach is that the structure of information defined by the DICOM SR is well known and can be understood through institutions with little effort. It is also important because we believe that DICOM SR will be widely adopted together with the CORBAmed specification.

The DICOM SR and the CIAS models were integrated by mapping the nodes of DICOM SR into Observations and the relationships of DICOM SR (HAS PROPERTY,CONTAINS, etc) to Observation Reference objects. For a more complete union of the standards the mapped information (in both directions) can be accessed by the Structured Reporting Object Model (SROM) which makes transparent the navigation in the tree nodes, supporting different implementations for the same interface. The union of these standards brings together the best side of each one, giving semantics to CIAS while improving DICOM through transparency of location, naming service and easier integration of legacy systems.
\end{abstract}

\section{Introduction}

During the last ten years an increasing amount of digital information has been generated and stored by healthcare providers. This information comes from reports, images, lab exams, patient demographics, financial data and digitized documents. The use of digital information helped to improve the services offered to the patients but it also increased the complexity of the systems used to generate and manage this information.

It is important to notice that hospitals have several information systems implemented in different periods of time from distinct vendors. These systems need to work together and exchange information, which is not a trivial task. Also, the higher complexity of the systems generates new demands for services like searching data, storage of new information and generation of reports. To solve these problems there is an effort to develop new standards and create reusable software solutions that allow different vendors to interact with each other in a comprehensive manner. Nowadays, the most adopted standards for medical information are DICOM (Digital Imaging and Communications in Medicine) and HL7 (Health Level 7). But those standards still miss in certain aspects that are needed nowadays to compose the Electronic Health Record, like security, object oriented design and integration of legacy systems.

There are several attempts to address these problems like HL7 version 3, new DICOM supplements and new approaches like Good Electronic Health Record (GEHR) [6] and CORBA in Medicine (CORBAmed) [7, 8].

The Healthcare Domain Task Force (also known as CORBAmed) is a common set of interfaces defined by the Object Management Group (OMG) for the access of medical information. Some of the services defined by CORBAmed are the Person Identification Service (PIDS), Clinical Observation Access Service (COAS) and Clinical Imaging Access Service (CIAS). The last one is the most interesting for implementation of PACS services and interchange of information between hospitals. But CORBAmed only specified the architecture to exchange information and not how (or what) content should be exchanged. This is a problem that needs to be addressed for the real implementation and adoption of the CORBAmed standard.

In this paper we propose an integration scheme of DICOM (more specifically DICOM SR) and CIAS that allow the building of a consistent framework for interchange of information and real interoperability.

\subsection{DICOM SR}

DICOM SR (DICOM Structured Reporting) is an addition to the DICOM standard [3,5] and provides a mechanism for encoding and interchanging of structured information.

DICOM SR represents a report as a node tree in a similar fashion to XML (Figure 1). This tree is restricted 
by a template that limits its possible nodes $[1,3,4]$.

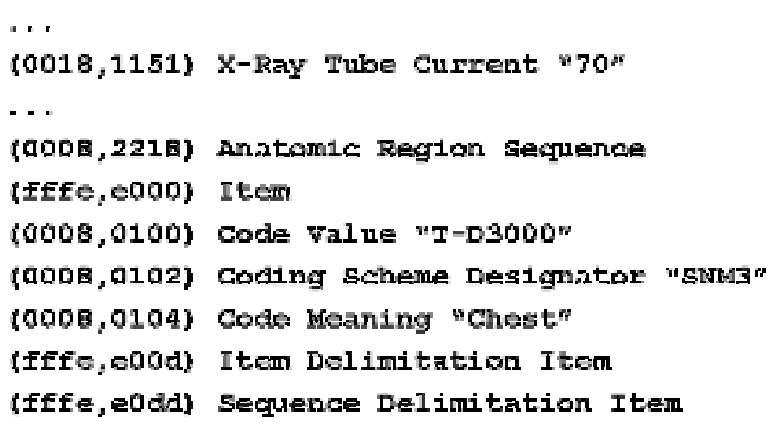

Figure 1. DICOM SR encoding.

The UML representation of the DICOM SR is shown in Figure 2 It can be noticed that every node has a relationship with his parent (represented in the model by relationshipType) that gives semantic to the structured information (for example, HAS PROPERTIES).

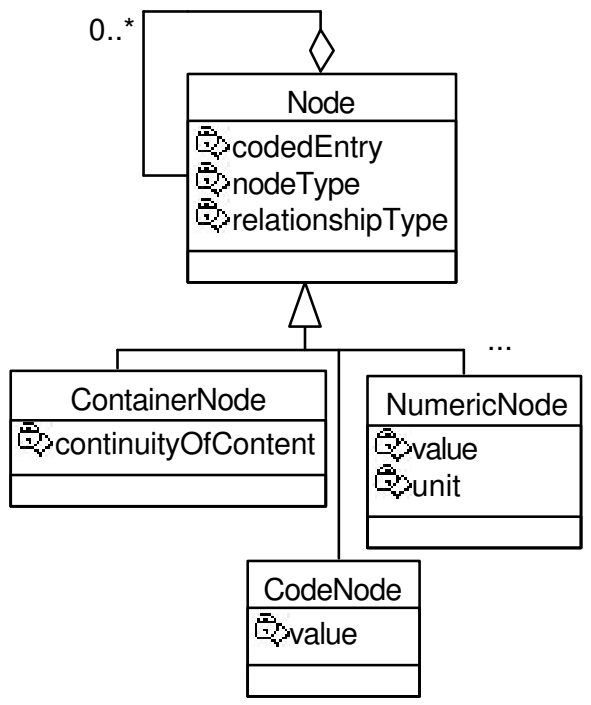

Figure 2. Tree representation of DICOM SR in UML

\subsection{CORBAmed}

Among the CORBAmed services, COAS (Clinical Observation Access Service) is the most generic because it is intended to allow the exchange of any kind of observation. Observations are defined by COAS as "any measurement, recording, or description of the anatomical, physiological, pathological, or psychological state or history of a human being or any sample from a human being, and any impressions, conclusions, or judgments made regarding that individual within the context of the current delivery of health care" [7].

The model adopted by COAS to represent an observation if shown in Figure 3. The information is organized in a hierarchic format composing a tree of observations. The nodes of the tree can be CompositeObservations or AtomicObservations. The former can only have children and no value while the latter can have a value but cannot have children.

Observations can be related to each other through an ObservationReference and an observation can have a qualifier (ObservationQualifier) that modifies the observation. Notice that a qualifier can have a value.

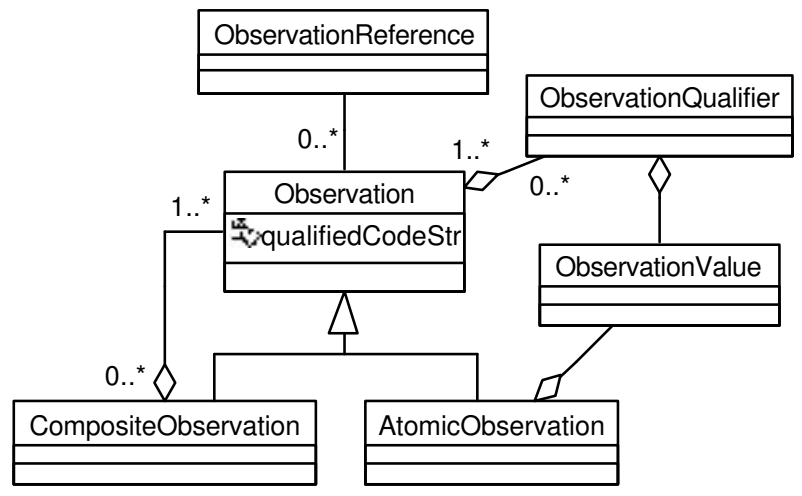

Figure 3. COAS observation model.

The CIAS service is an extension of the COAS service, including search for medical images and the same model of observations from COAS can be applied to CIAS.

\subsection{SROM}

SROM (Structured Reporting Object Model) is described in [1] and is a set of common interfaces to access structure and content of the SR tree regardless of its internal or external representation [5].

\section{Joining DICOM SR and CIAS}

Both DICOM SR and CIAS represent information as a directed acyclic graph but with some differences: in DICOM SR all the nodes can have children while in CIAS only a CompositeObservation is allowed to have children (see Figures 2 and 3). At the same time the CompositeObservation cannot have a value, which is only allowed to an AtomicObservation. The solution adopted was to define all the nodes as CompositeObservations with a child that is an AtomicObservation containing the real value of the node. Table 1 shows the QualifiedCodeStrings created and the value types of the AtomicObservations. 
Table 1. Mapping nodes from DICOM SR to COAS.

\begin{tabular}{|c|c|c|}
\hline DICOM & COAS & \\
\hline Node Type & QualifiedCodeStr & Value \\
\hline Container & $\begin{array}{l}\text { InCor/DICOMSR/valueType/ } \\
\text { Container }\end{array}$ & Plain Text \\
\hline Text & $\begin{array}{l}\text { InCor/DICOMSR/valueType/ } \\
\text { Text }\end{array}$ & Plain Text \\
\hline Code & $\begin{array}{l}\text { InCor/DICOMSR/valueType/ } \\
\text { Code }\end{array}$ & $\begin{array}{l}\text { Coded } \\
\text { Element }\end{array}$ \\
\hline Num & $\begin{array}{l}\text { InCor/DICOMSR/valueType/ } \\
\text { Num }\end{array}$ & Numeric \\
\hline PName & $\begin{array}{l}\text { InCor/DICOMSR/valueType/ } \\
\text { PName }\end{array}$ & Plain Text \\
\hline Date & $\begin{array}{l}\text { InCor/DICOMSR/valueType/ } \\
\text { Date }\end{array}$ & Date Time \\
\hline Time & $\begin{array}{l}\text { InCor/DICOMSR/valueType/ } \\
\text { Time }\end{array}$ & Date Time \\
\hline DateTime & $\begin{array}{l}\text { InCor/DICOMSR/valueType/ } \\
\text { DateTime }\end{array}$ & Date Time \\
\hline UidRef & $\begin{array}{l}\text { InCor/DICOMSR/valueType/ } \\
\text { UIDRef }\end{array}$ & $\begin{array}{l}\text { Coded } \\
\text { Element }\end{array}$ \\
\hline Composite & $\begin{array}{l}\text { InCor/DICOMSR/valueType/ } \\
\text { Composite }\end{array}$ & $\begin{array}{l}\text { Composite } \\
\qquad(*)\end{array}$ \\
\hline Image & $\begin{array}{l}\text { InCor/DICOMSR/valueType/ } \\
\text { Image }\end{array}$ & $\underset{(*)}{\text { Image }}$ \\
\hline Waveform & $\begin{array}{l}\text { InCor/DICOMSR/valueType/ } \\
\text { Waveform }\end{array}$ & $\begin{array}{c}\text { Waveform } \\
(*)\end{array}$ \\
\hline SCoord & $\begin{array}{l}\text { InCor/DICOMSR/valueType/ } \\
\text { SCoord }\end{array}$ & $\begin{array}{l}\text { SCoord } \\
(*)\end{array}$ \\
\hline TCoord & $\begin{array}{l}\text { InCor/DICOMSR/valueType/ } \\
\text { TCoord }\end{array}$ & $\begin{array}{l}\text { TCoord } \\
(*)\end{array}$ \\
\hline Node & InCor/DICOMSR/Node & $(* *)$ \\
\hline
\end{tabular}

(*)ObservationValues created specifically for DICOM SR

(**) Represents the Node as a CompositeObservation.

The relationships were mapped as shown in Table 2 .

Using these mappings and the SROM interface it is possible to have a common interface for both DICOM SR and COAS observations. This makes transparent for the user the access to information, eliminating one of the problems of COAS that is how to interpret the transmitted information.

Figure 4 on the next page shows an instance diagram illustrating how the mapping can be done.
Table 2. Mapping relationships from DICOM SR to COAS

\begin{tabular}{ll}
\hline RelationshipType & QualifiedCodeStr \\
\hline CONTAINS & $\begin{array}{l}\text { InCor/DICOMSR/relation/ } \\
\text { Contains }\end{array}$ \\
\hline HAS PROPERTIES & $\begin{array}{l}\text { InCor/re lation/DICOMSR/ } \\
\text { Has_Properties }\end{array}$ \\
\hline INFERRED FROM & $\begin{array}{l}\text { InCor/relation/DICOMSR/ } \\
\text { Inferred_From }\end{array}$ \\
\hline SELECTED FROM & InCor/relation/DICOMSR/ \\
& Selected_From \\
\hline HAS OBS CONTEXT & InCor/relation/DICOMSR/ \\
& Has_Obs_Context \\
\hline HAS ACQ CONTEXT & InCor/relation/DICOMSR/ \\
& Has_Acq_Context \\
\hline HAS CONCEPT MOD & InCor/relation/DICOMSR/ \\
& Has_Concept_Mod \\
\hline
\end{tabular}

\section{Final remarks}

DICOM is a specific standard for medical images that is now addressing the (difficult) problem of encoding reports. The inclusion of Structured Reporting in DICOM provides [9]:

- $\quad$ semantic documentation of diagnosis ;

- context to a report;

- $\quad$ linking of text with images, waveforms, audio and measurements;

- flexibility enough to go beyond radiology imaging;

- facility for computer analysis.

Furthermore, DICOM is a largely adopted standard, supported for several vendors that, we believe, will quickly incorporate DICOM SR.

DICOM presents, however, some weaknesses when we are dealing with the exchange of information between healthcare providers, like security and the capacity to perform complex queries. Also the access to legacy systems and integration of heterogeneous distributed information can be difficult to achieve.

On the other hand, by using CORBA and its specification in healthcare (CORBAmed) these goals can be achieved.

The integration of CORBA and DICOM SR can facilitate the development of information systems for healthcare, providing a reliable framework for building a coherent and flexible system that can serve current and future demands in healthcare.

The union of these standards brings together the best side of each one, giving semantics to CIAS while improving DICOM through transparency of location, naming service and easier integration of legacy systems . 


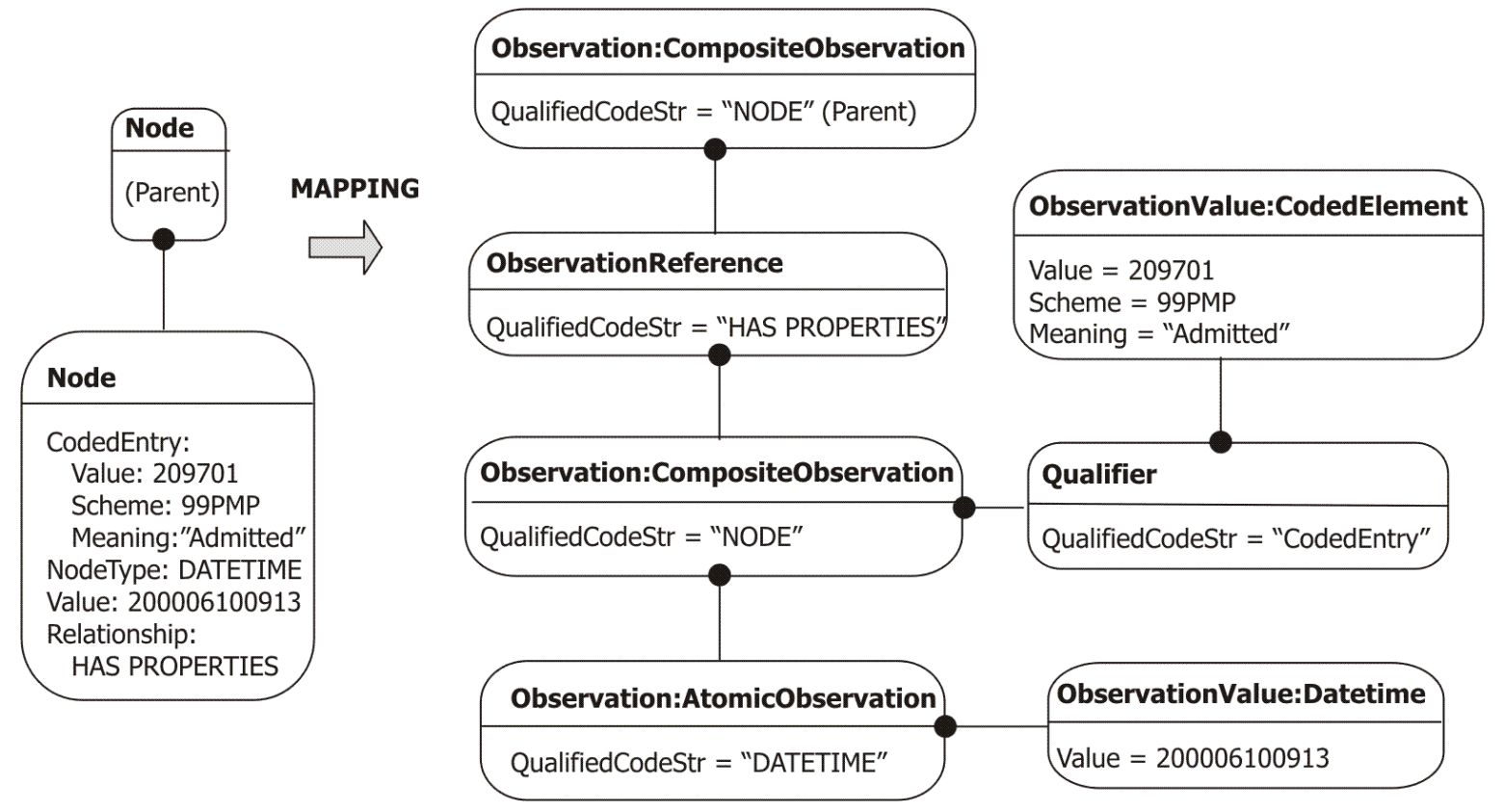

Figure 4. Instance diagram of mapping from DICOM SR to COAS.

\section{Acknowledgements}

This project has been supported by FAPESP grants 1997/14206-5 and 2001/05987-0, the Zerbini Fundation and the Polytechnic School of São Paulo (EPUSP).

\section{References}

[1] CLUNIE DA. DICOM Structured Reporting. PixelMed Publishing, 2000. Available at http://www.pixelmed.com/ srbook.html.

[2] ACR-NEMA, "Digital Imaging and Communications in Medicine - PS 3.3", National Electrical Manufacturers Association, 2000.

[3] ACR-NEMA, "Digital Imaging and Communications in Medicine - Supplement 23: Structured Reporting Storage SOP Classes", National Electrical Manufacturers Association, 2000.

[4] ACR-NEMA, "Digital Imaging and Communications in Medicine - Supplement 53: DICOM Content Mapping Resource", National Electrical Manufacturers Association, 2001.
[5] CLUNIE DA. 'DICOM Structured Reporting: An object model as an implementation boundary". Proc. of SPIE, San Diego, 2001.

[6] The Good Electronic Health Record Web Site. Accessible at http://www.gehr.org/.

[7] Clinical Observation Access Service Specification. Available at www.omg.org/cgi-bin/doc?formal/2001-04-06.

[8] Clinical Image Access Service Specification. Available at http://cgi.omg.org/docs/dtc/01-08-01.pdf.

[9] SYCLE, DEV, "DICOM Structured Reporting: Understand Basic Text and Enhanced SR Content", Merge Technologies Inc. Available at http://www.rsna.org/IHE/ iheyr2wkshp/iheyr2_dicom-sr-content.pdf.

Address for correspondence.

Ramon Alfredo Moreno.

Instituto do Coração (InCor) HC.FMUSP

Av. Dr. Enéas de Carvalho Aguiar, 44, Div. de Informática, $2^{\circ}$ andar, CEP 05403-000, São Paulo, SP, Brazil. ramon.moreno@incor.usp.br. 